\title{
Forced Inefficiencies of the Electronic Health Record
}

\author{
Michael Weiner, $M D, M P H^{1,2,3}$
}

${ }^{1}$ Center for Health Information and Communication, U.S. Department of Veterans Affairs, Veterans Health Administration, Health Services Research and Development Service CIN 13-416, Richard L. Roudebush VA Medical Center, Indianapolis, IN, USA; ${ }^{2}$ Regenstrief Institute, Inc., Indiana University School of Medicine, Indianapolis, IN, USA; ${ }^{3}$ Indiana University Center for Health Services and Outcomes Research, Indianapolis, IN, USA.

J Gen Intern Med 34(11):2299-301

DOI: $10.1007 / \mathrm{s} 11606-019-05281-3$

(c) Society of General Internal Medicine (This is a U.S. government work and not under copyright protection in the U.S.; foreign copyright protection may apply) 2019

$\mathrm{T}$ he medical record has been a subject of science, and an object of intervention, for decades, but at times, it still seems that our progress misses the mark - not just someone else's mark, but even our own. We want today's electronic health record (EHR) systems to serve as efficient tools to document and even facilitate effective care of individual patients and populations. Although the EHR has transformed the medical record from simply a historical record into more of a "living document" - as writers can tag each other with specific questions or notes, or have referrals electronically sent from one point to another, for example - it has also continued to enable inconsistency and potentially undesirable variation in documentation and, sometimes, even the clinical care associated with it.

In this issue, Cohen et al. advance our knowledge about this variation in documentation, by reporting a large EHR-based study and discussing variation's potential to harm patients. ${ }^{1}$ Using EHR logs of 809 physicians, and interviews with 40 outpatient physicians, they assessed the proportion of encounters in which healthcare professionals completed documentation of specific types. They found that five types of documentation had significant variation at the physician level, independent of state, organization, and practice: written discussion of clinical results (78\% of variation attributed to physicians), assessment and diagnosis (76\%), problem list (70\%), review of systems $(68 \%)$, and social history $(62 \%)$. Among these types, the interquartile range of the proportion of encounters with completed documentation ranged from 51 to $73 \%$. Interviewees noted issues such as multiple places to record the same information. As the authors indicated, variation might refer to content, structure, or location of information; furthermore, variation within practices, where patient mix might be relatively uniform at least compared with other practices, may relate most to physicians' individual preferences and personal styles in documentation. The study's attention to teasing apart the sources of variation, such as the physician, and the types and places of variation in the medical record, is commendable and informative. This research advances what we know about variation, both in its quantitative extent and in participants'

Published online August 26, 2019 perceptions about it. Cohen et al. have suggested that, for the benefit of our patients, we need a greater degree of standardization or consistency in documentation.

As the authors note, some studies of variation and quality of documentation have been done. Linder et al. reported that dictated notes were associated with lower quality of care, compared with directly typed notes. ${ }^{2}$ Primary care providers have especially favored templates, rather than more narrative methods of generating documentation. ${ }^{3}$ Ancker et al. found that the proportion of encounters with updated problem lists ranged from 5 to $60 \%$ per provider per year, with updates most likely for new patients rather than established ones. ${ }^{4}$ Edwards et al. reported that medical records often lacked even the most basic elements, such as reason for visit (missing in $10 \%$ of their sample), medication lists (20\% missing), and planned timing of follow-up (18\% missing). ${ }^{5}$

With clinicians spending about half of their day on EHR and desk work, ${ }^{6}$ how will we translate the many findings from research about documentation into better clinical practice? How will we rein in the "wild west" of EHR authorship, and stop misplacement of information, burnout related to documentation burdens, ${ }^{7}$ and other adverse consequences of EHR design? The authors mentioned potential strategies such as user training, and practice sessions focusing on standardization of documentation. Structured documentation workflows, including embedded training practices, have been used in pharmacy practice and found to be beneficial. ${ }^{8}$ Although experience and research ${ }^{9}$ tell us that training and practice are important, these are probably still not sufficient strategies. The work force changes rapidly, many individual clinicians use multiple different EHR systems, and practice requires much time. The biggest problem with suggesting training and practice as primary solutions is that these sometimes represent workarounds to failed EHR designs: instead of using the power of human-factors engineering-used in all of today's automobiles and aircraft - we buy EHR products of any type (though with limited choices), and then, immediately on day one, start designing and even teaching strategies to overcome their significant limitations. As noted, these often-compensatory strategies might compromise the safety of care, though Cohen's study itself was not designed to measure safety.

Some flexibility in documentation practice appears to be helpful and important. Rosenbloom et al. have eloquently discussed many of the important nuances and implications of documentation, including the tension between structure and 
flexible documentation. ${ }^{10}$ Nonetheless, we need to be able to use and reuse the medical record efficiently. Indeed, as Rosenbloom's team indicated, generating reusable data is a major goal of integrating clinical documentation into the EHR. One way to help with the problem of variation is to make better use of automation that handles the widely varying sections of documentation for us. For example, why would we need to update problem lists manually, when EHR users already document important clinical problems at the end of every note? An advanced EHR system should find, assemble, and maintain its own problem list automatically, based on those notes. Some systems do this to some extent, by automatically adding problems from notes. Text processing can be used to produce structured data, and to map text to standardized concepts using defined terminologies. ${ }^{10}$ The part of managing a problem list that is perhaps even more difficult than building it is deciding when a problem has been resolved or is no longer important, and should be removed from the list. We need EHR systems that can find, organize, and appropriately build and purge listings of problems, automatically. A second case is the review of systems. Patients can, and should, directly generate their own review of systems, which is populated in the record and then addressed by the healthcare team. Some institutions have started to do that - "patient-generated health data" is one of the terms - but we have a long way to go towards better integration and effective uses of this type of information. ${ }^{11}$

The now-familiar copy-and-paste phenomenon is an example of how a certain type of lack of variation can paralyze us. When we see exactly the same information again and again, it can be even worse than having to find things in hidden places. Edwards's team found pasted material in more than $10 \%$ of notes. ${ }^{5}$ Another group examining ophthalmology notes with a mean of 1182 words found that less than a quarter of the words changed between visits. ${ }^{12}$ When variation in documentation occurs and makes information difficult to find, the healthcare team takes up the slack: many healthcare professionals do not skip the task of finding the information, but instead, search harder, and spend more time looking for what they need. As such, we have become "the invisible fixers". The fixers are each of us - the people who will inefficiently work to solve the clinical problem at hand, because our workaround appears to be the only way to solve it now, today, at the point of care. This work is often unnoticed, undervalued, and unaddressed, and may even contribute to the kinds of variation that Cohen et al. have discovered. We need to stop ignoring the forced inefficiencies and instead make them the spotlight of our attention. Responsibility for documentation is shared by EHR vendors, administrators of health institutions, and front-line users. Along the way, we should be sure to question the usefulness of some of our traditions: is a review of systems important and, if so, how should we best capture and use the information - perhaps differently than we have always done?

Cohen's study is thought-provoking about how to solve the problem of variation, and even understanding when it might not be a problem. A litany of questions comes to mind. Does the specific EHR system matter? What elements of social history really need to be reassessed upon every visit? When does variation cause inefficiency, and when is it an effect of inefficiency? When will standardizing documentation overcompensate for the problem? When is a checkbox better than a narrative? How and when is team documentation most effectively used? How can touchscreens, voice recognition technologies, mind-based controls, and similar technologies, be used to speed data entry, which is the slowest part of using EHR systems? How does EHR-mediated harm occur? We need answers. In the meantime, below, I suggest several principles of documentation relating to variation. Many of these target EHR vendors, but some also target EHR users, trainers, and others working in healthcare settings.

- Use checkboxes or short-answer form fields when questions have answer options that are few, discrete, and structured. This may help to decrease errors, improve analyzability, and decrease time requirements.

- Foster narrative text when it may be most useful: the history of the present illness, and assessment of findings. This can help to maximize richness of detail and communication of thoughts that are important in making diagnoses and medical decisions.

- Avoid promoting repetition within a single note. For example, automatically extract problems and diagnoses from assessments and plans, instead of requiring the user to document each problem twice. This could improve overall efficiency, decrease errors, and decrease time requirements.

- Minimize requirements for training and practice, by making the system intuitively easy to use and understand, to the greatest extent possible. This could decrease time requirements and incidence of errors.

- Foster similarities of beneficial approaches across disparate medical-record systems, to improve consistency and decrease errors that may otherwise be caused by required variation in usage by system.

- Create innovative ways to update standing lists, so that they are periodically and perhaps semi-automatically purged of useless information.

- Include anticipatory guidance in automated recommendations. For example, if a patient has hypothyroidism, I might want to view or order the thyroid function tests, right? Help me get that done before I have to think of it and do it myself.

- Group related information together.

- Make searching for information within a record or across records as easy as it is on the rest of the computer.

- Foster patients' involvement in generating parts of the data, such as the review of systems, and current medication lists.

- Use strategies that are thought or shown to save time, such as minimizing the number of clicks, providing 
options for keyboard shortcuts, and making touchscreens available for selected functions.

- Automate the billing-based coding of encounters, because that coding is based entirely on documentation. This will save time and may improve accuracy and consistency of coding, compared with manual coding.

- Incorporate synchronous communication technologies into the software that maintains the medical record. For example, if I need to call the on-call cardiologist about my patient, tell me who it is, and help me contact that person here and now.

Most of all, when it comes to the record and me, I still want my voice, that part of the record that tells other people what I think the problem is, and what should be done about it. Variation or not, that is the most important part of the medical record.

Corresponding Author: Michael Weiner, MD, MPH; Regenstrief Institute, Inc. Indiana University School of Medicine, Indianapolis, IN, USA (e-mail: mw@cogit.net).

Funding Information The research reported here was financially supported by the US Department of Veterans Affairs, Veterans Health Administration, Health Services Research and Development Service CIN 13-416.

\section{Compliance with Ethical Standards:}

Conflict of Interest: Dr. Weiner is Chief of Health Services Research and Development at the Richard L. Roudebush Veterans Affairs Medical Center in Indianapolis, IN.

Disclaimer: The views expressed in this article are those of the authors and do not necessarily represent the views of the US Department of Veterans Affairs.

\section{REFERENCES}

1. Cohen GR, Friedman CP, Ryan AM, Richardson CR, Adler-Milstein J. Variation in Physicians' Electronic Health Record Documentation and Potential Patient Harm from That Variation. J Gen Intern Med. 2019. https://doi.org/10.1007/s11606-019-05025-3

2. Linder JA, Schnipper JL, Middleton B. Method of electronic health record documentation and quality of primary care. J Am Med Inform Assoc. 2012;19(6):1019-24. https://doi.org/10.1136/amiajnl-2011000788

3. Pollard SE, et al. How physicians document outpatient visit notes in an electronic health record. Int J Med Inform. 2013;82(1):39-46. https:// doi.org/10.1016/j.ijmedinf.2012.04.002

4. Ancker JS, et al. How is the electronic health record being used? Use of EHR data to assess physician-level variability in technology use. J Am Med Inform Assoc. 2014;21(6):1001-8. https://doi.org/10.1136/ amiajnl-2013-002627

5. Edwards ST, Neri PM, Volk LA, Schiff GD, Bates DW. Association of note quality and quality of care: a cross-sectional study. BMJ Qual Saf. 2014;23(5):406-13. https://doi.org/10.1136/bmjqs-2013-002194

6. Sinsky C, et al. Allocation of Physician Time in Ambulatory Practice: A Time and Motion Study in 4 Specialties. Ann Intern Med. 2016;165(11):753-60. https://doi.org/10.7326/M16-0961

7. Flanagan ME, Militello LG, Rattray NA, Cottingham AH, Frankel RM. The Thrill Is Gone: Burdensome Electronic Documentation Takes Its Toll on Physicians' Time and Attention. J Gen Intern Med. 2019. https://doi. org/10.1007/s11606-019-04898-8

8. McNicol M, Kuhn C, Sebastian S. Standardized documentation workflow within an electronic health record to track pharmacists' interventions in pediatric ambulatory care clinics. J Am Pharm Assoc (2003). 2019;59(3):410-5. https://doi.org/10.1016/j.japh.2019.01.007

9. Ventres W, Kooienga S, Vuckovic N, Marlin R, Nygren P, Stewart V. Physicians, Patients, and the Electronic Health Record: An Ethnographic Analysis. Ann Fam Med. 2006;4(2):124-31. https://doi.org/10.1370/ afm. 425

10. Rosenbloom ST, Denny JC, Xu H, Lorenzi N, Stead WW, Johnson KB. Data from clinical notes: a perspective on the tension between structure and flexible documentation. J Am Med Inform Assoc. 2011;18(2):181-6. https://doi.org/10.1136/jamia.2010.007237

11. Hendrickson MA, Melton GB, Pitt MB. The Review of Systems, the Electronic Health Record, and Billing. JAMA. 2019. https://doi.org/10. 1001/jama.2019.5667

12. Huang AE, Hribar MR, Goldstein IH, Henriksen B, Lin W-C, Chiang MF. Clinical Documentation in Electronic Health Record Systems: Analysis of Similarity in Progress Notes from Consecutive Outpatient Ophthalmology Encounters. AMIA Annu Symp Proc. 2018;20181310-8.

Publisher's Note Springer Nature remains neutral with regard to jurisdictional claims in published maps and institutional affiliations. 